\title{
A Cone Rotating in a Fluid Translates
}

\section{Kern E. Kenyon}

4632 North Lane, Del Mar, USA

Correspondence to: Kern E. Kenyon, kernken@aol.com

Keywords: Rotating Cone in a Fluid, Rotating Cone Translating in a Fluid

Received: January 31, $2020 \quad$ Accepted: February 25, $2020 \quad$ Published: February 28, 2020

Copyright $\odot 2020$ by author(s) and Scientific Research Publishing Inc.

This work is licensed under the Creative Commons Attribution International License (CC BY 4.0).

http://creativecommons.org/licenses/by/4.0/

\section{(c) (i) Open Access}

\section{ABSTRACT}

When a solid cone with smooth surfaces rotates at a constant rate about its long axis in still water, it will experience no friction, as announced earlier [1] and documented further here. However, if not restrained, it will translate along the axis base first and apex last, which is caused by a variation of pressure on its side: low near the base and high at the apex. This translation needs to be verified experimentally. Friction will occur during translation but an opposite reaction force will also take place related to the front to back asymmetry of the body [2]. Whether or not these two oppositely directed forces can cancel each other out by an appropriate choice of variable magnitudes is not known, but if true, it would lead to an extended translation. Also the translation path could be lengthened by slightly modifying the front face of the cone such that the translation itself produces rotation. One idea for such a modification is suggested. Observations in the future would be very beneficial.

\section{INTRODUCTION}

Contained in the title is an idea briefly proposed recently [1]. More discussion here brings focus to the theory in addition to some unavoidable technical complications. It is well known that a certain class of apparatus, like a windmill, fixed in position, will rotate when the wind blows, and the greater the wind speed, the faster the rotation rate is. A solid cone rotating in a fluid like water should feel the pull to translate along the axis of rotation, base first and apex last. And the faster the rotation the greater the pull is to translate. What might happen if these two separate ideas were attempted to be coupled together? A few options are offered below.

Initially the rotating/translating cone idea did not come from observing nature, which has sometimes happened in the past through the stumbling process of evolution. A horn shark egg case led me down the wrong path for a short period. It is roughly cone shaped but after a couple of weeks in the water the distinctive spiral vane becomes vertical to the cone's surface and stiffens. Surely such an unusual shape must have occurred for a reason. At first I thought the reason was to make the egg rotate as it translated. However, by pushing a couple of these eggs by hand through the water in an aquarium tank, I found out that the reaction I was expecting did not happen. Rotation stopped abruptly after one or two revolutions. Crit- 
ical to the present concept is a smooth surface of the cone's side.

An adaptation of a theory published in a familiar fluid dynamics text [3] produced the result. When a solid cylinder rotates at a constant rate in a still fluid, there is no friction in the fluid and none experienced by the cylinder. That much is amazing by itself. In the earlier exposition [1] the assumption was made that there would also be no friction if a solid cone rotated in a fluid. But that assumption does not need to be made, because it can be verified by consulting the Navier-Stokes equations in cylindrical polar coordinates (instead of plane polar coordinates for the rotating cylinder), since the variation of the rotational velocity component is linear along the cone's surface normal to the flow direction [4]. The other two velocity components follow the cylinder's example.

Inherent in the earlier qualitative construction of the pressure force on the rotating cone's surface driving the translation is the application of Bernoulli's law to the steady flow in closed circular streamlines surrounding the cone. This also is not an assumption but a theory that already has seen a few applications [5]. And an independent theory in the fluid dynamics text [3] gives the same results for the rotating cylinder, such as the fall off rate of the perturbed velocity away from the solid body (see Equation (1)), which however does not make use of Bernoulli's law, so the two separate theories agree with each other. According to Bernoulli's law the pressure is least where the speed is greatest. Thus the pressure on the cone's surface near the base is lower than at the apex. That is how the notion of translation comes about.

Observations have not yet verified the forecasted translation of a rotating cone. Nevertheless, a broadening of the theory is thought to be useful to present next.

\section{EXTENSION}

In all planes perpendicular to the cone's axis of rotation, Bernoulli's law is applied to the closed streamlines of fluid flowing around the cone. Also the cross-stream force balance between the outward centrifugal force and the inward pressure force is used. When the pressure is eliminated between these two equations, the result for the velocity $v$ is

$$
\frac{\mathrm{d} v}{\mathrm{~d} r}=-\frac{v}{r}
$$

where $r$ is the radial coordinate measured from the axis of rotation. Equation (1) is a linear differential equation with the solution: $v=$ const $/ r$ [5], which has no associated friction.

Hypothetically consider a cone under water starting from zero to rotate at a constant rate by some unspecified mechanism. After a spin-up time the initial velocity stuck to the cone's surface by friction will be communicated outward until the $1 / r$ fall off rate in the adjacent fluid is established, and then all friction stops. At that point the rotation mechanism can be disconnected and the cone will keep on rotating. During this start up process there will be developed a pressure force on the side of the cylinder attempting to make the cone translate. Suppose this is prevented from happening initially until equilibrium in the fluid flow sets in. Then the holding back part is released. What happens next?

For simplicity or reasons of "aerodynamics" let the flat base of the cone become a lens slightly bulging forward (smaller than a half sphere) and the joint between lens and cone made smooth to avoid turbulence. Flow past the lens/cone will occur and begin to interact with the flow pattern already set up by the rotation. This is one of the complications mentioned above which puts a strain on the imagination.

Take a step back to first consider the known behavior of a non-rotating lens/cone moving in a still fluid. In this case there is friction tending to slow down the solid body's advance. But there is also a net reaction force in the opposite direction due to Newton's third law and to fluid accelerations and decelerations along the surface of this solid body with front to back asymmetry [2] (there is axial symmetry). So far it has not been possible to compare the magnitudes of these two oppositely directed forces for such a solid body because of a lack of information about the laminar friction force. Nature did discover this reaction force before man did and fish, for example, use it to advantage with their blunt front approach to forward movement. 
Onward again to rotation. As the flow passes over the surface of the rotating lens on its way to the rotating cone, a relatively small amount of friction will take place there. Since the rotating cone surface already has in place an attached boundary layer set up, the flow going by will probably have to go around at least the outer weaker part of it. That is an assumption. A little distance from the cone's surface the flow passing by and the boundary layer flow will interact such that there will be friction between them. But the cone's surface is shielded from that effect. The inter-flow friction may be smaller than if the rotary boundary layer were not present. In any case, that frictional effect will not reach the cone to slow down the translational speed. And to counteract the friction between the two flows will be a reaction force due to the asymmetric accelerations and decelerations along the curving streamlines from front to back. If the magnitudes of these two oppositely directed forces could ever exactly cancel each other out is not known at the present time.

\section{CONCLUSION}

Once set in place as outlined hypothetically above, the translating and rotating cone could travel some distance on its own until the relatively small net amount of friction ultimately slows down the rotation, which then stops the translation. What if translation by itself could cause rotation? For example, if a couple of small slanted vanes, perpendicular to the surface, were to be attached to the lens, then flow due to translation passing by would cause rotation of the whole solid body. Adjustment of the size and angle of slanting of the vanes could be done to optimize the results. Also more than two vanes might work better. Add in the forward directed reaction force, induced in the flow by the front to back asymmetry of the cone, to counteract the friction of the flow passing by the cone and interacting with the boundary layer flow stuck to the rotating surface. Is perpetual motion a possibility, or is this just a ridiculous suggestion? Were any assumptions made above not valid? Observations would help a great deal obviously; difficulties in doing so are not anticipated to be insurmountable.

\section{CONFLICTS OF INTEREST}

The author declares no conflicts of interest regarding the publication of this paper.

\section{REFERENCES}

1. Kenyon, K.E. (2020) Cone Rotating in a Fluid. Natural Science, 12, 1-3. https://doi.org/10.4236/ns.2020.121001

2. Kenyon, K.E. (2017) Asymmetric Solids Move Faster in Water When the Blunter end Leads. European International Journal of Science and Technology, 6, 61.

3. Batchelor, G.K. (1967) An Introduction to Fluid Dynamics. Cambridge University Press, San Diego, 203.

4. Kundu, P.K. (1977) Fluid Mechanics. Academic Press, San Diego, 622.

5. Kenyon, K.E. (2019) Bernoulli Loops with No Friction. European International Journal of Science and Technology, 8, 25-28. 